\title{
Correction of Accurate Mass Measurement for Target Compound Verification by Quadrupole Time-of-flight Mass Spectrometry
}

\author{
Harald C. Köfeler and Michael L. Gross \\ Department of Chemistry, Washington University, St. Louis, Missouri, USA
}

\begin{abstract}
The aim of this work is to evaluate quadrupole/time-of-flight (QTOF) mass spectrometry for simultaneous measurement of accurate mass and quantification of a target by using a stable isotopically labeled internal standard. Mixtures of caffeine and ${ }^{13} \mathrm{C}_{3}$-caffeine (internal standard) at different concentration ratios were analyzed by capillary HPLC/QTOF. A calibration plot for quantification is linear over a factor of 20 . Without invoking any correction scheme, the mass accuracy seriously degraded when the ratio of the mass standard to the test compound was not unity. The accuracy could be restored to approximately $2 \mathrm{ppm}$ by using a quadratic function to correct the measured mass as a function of the measured signal ratio of target and internal calibrant. (J Am Soc Mass Spectrom 2005, 16, 406-408) (c) 2004 American Society for Mass Spectrometry
\end{abstract}

$\mathrm{A}$ lthough accurate mass measurement, usually at high mass resolving power, is commonly used to identify new compounds, it can also add certainty to the detection of a target, particularly in trace analysis. The classic example is the trace analysis of various chlorinated dioxins and dibenzofurans by GC/MS [1], a trace method that was validated over 20 years ago [2, 3]. High resolving power also allows interferences to be excluded, especially when peak profiles are obtained $[1,4]$. MS/MS is another means to achieve specificity, and the choice between it and accurate mass is one of the tradeoffs. Although a peak obtained at low resolving power can also give accurate mass, detection suffers because unresolved interferences can cause confusion [3,5].

The use of electrospray ionization (ESI) in combination with liquid chromatography (LC) has expanded target-compound analysis to biologically important compounds. Fast-scanning time-of-flight (TOF) and Fourier transform mass analyzers, with their good resolving power, accurate mass, and peak profiles, should afford specificity in LC/MS. Although FTMS has higher mass accuracy and resolving power [6, 7], FT-based instruments are not as accessible as those that are TOF-based.

The orthogonal acceleration TOF mass analyzers

Published online January 29, 2005

Address reprint requests to Dr. M. L. Gross, Department of Chemistry, Washington University, One Brookings Drive, St. Louis, MO 63130, USA. E-mail: neff@wuchem.wustl.edu offer fast scanning and medium mass resolving power. Their mass measurement precision/accuracy can be \pm 5 ppm when a mass calibrant $[8,9]$ is cointroduced by various methods [10-12]. When interfaced to a quadrupole (QTOF), accurate mass measurement in the MS/MS mode is even possible [8]. Because these instruments commonly employ ion counting, mass accuracy is best when counts are low and peak shapes are undistorted. As for other instruments, the accuracy is best when the $m / z$ of the unknown and the mass calibrant are nearby [8].

The motivation for this work is to evaluate the use of a chromatographically coeluting internal standard in LC/MS as a calibrant for accurate mass measurement with a Q-TOF instrument. Although others have shown that mass-measurement errors increase as ion counts for analyte and calibrant begin to differ [9], we wish to explore the extent of the variation and to test whether it can be corrected.

\section{Experimental}

Analytical grade methanol, acetic acid, and caffeine were from Sigma (St. Louis, MO). ${ }^{13} \mathrm{C}_{3}$-caffeine was from ISOTEC, Miamisburg, OH.

The experiments were performed using a Q-TOF Ultima Global (Micromass, Manchester, UK) hybrid mass spectrometer equipped with an orthogonal electrospray source (Z-Spray) operated at $3 \mathrm{kV}$ and controlled by MassLynx 4.0. The source temperature was $100{ }^{\circ} \mathrm{C}$, and the desolvation temperature $200^{\circ} \mathrm{C}$. The 
TOF was operated at an acceleration voltage of $9.1 \mathrm{kV}$, a cone voltage of $100 \mathrm{~V}$, and a collision energy of $10 \mathrm{eV}$. The mass spectrometer was coupled to a Waters CapLC system, operated at a flow rate of $7 \mu \mathrm{L} / \mathrm{min}$. A ThermoHypersil C-18 column $(150 \times 0.32 \mathrm{~mm} \mathrm{5- \mu \textrm {m } 1 0 0 - \AA ̊ )})$ was used with a gradient: Solvent A (water/methanol 97:3; $0.05 \%$ acetic acid) was held at $100 \%$ for $3 \mathrm{~min}$, decreased to $10 \%$ in $2 \mathrm{~min}$, and slowly decreased to $5 \%$ for $10 \mathrm{~min}$. Solvent B was methanol with $0.05 \%$ acetic acid. The injection volume was $5 \mu \mathrm{L}$. A mass range, $m / z$ 193-201, was scanned continuously over the chromatographic run at a rate of 1 spectrum/s, acquiring data for analyte and calibrant in one spectrum. The mass spectrometer was tuned and calibrated with a 1:1 mixture of caffeine and ${ }^{13} \mathrm{C}_{3}$-caffeine. All measurements were in triplicate.

\section{Results and Discussion}

We chose caffeine as analyte and its isotopomer as mass standard to evaluate the measured mass accuracy of a QTOF for target compound analysis.

Caffeine $(\mathrm{m} / \mathrm{z} 195.0882)$ and ${ }^{13} \mathrm{C}_{3}$-caffeine $(\mathrm{m} / \mathrm{z}$ 198.0951) elute at the same retention time, which was stable at $10.04 \pm 0.09 \mathrm{~min}$, with a chromatographic peak width of approximately $6 \mathrm{~s}$ full width half maximum. To establish a quantitative calibration plot, we held the concentration of ${ }^{13} \mathrm{C}_{3}$-caffeine constant at $100 \mathrm{nM}(500 \mathrm{fmol}$ on column) and varied that of caffeine from $25 \mathrm{nM}$ (125 fmol) to $500 \mathrm{nM}(2.5 \mathrm{pmol})$. The calibration curve shows linearity in the high concentration range, but at lower concentrations, signal intensities level off. Nevertheless, a fit shows good linearity over the concentration range $\left(R^{2}=0.9985\right)$ and a limit of detection of $10 \mathrm{nM}(50 \mathrm{fmol}, \mathrm{S} / \mathrm{N}=3)$. At a concentration of $500 \mathrm{nM}$, the signal ratio of target and internal standard levels off, indicating the upper limit of the linear range. This behavior suggests the MCP is being overloaded.

Although the accuracy of mass measurement on a Q-TOF should be $\sim 5 \mathrm{ppm}$ with an internal calibrant, we found the accuracy depends significantly on the signal intensity ${ }^{\circ}$ ratio $^{\circ}$ for ${ }^{\circ}$ the ${ }^{\circ}$ target ${ }^{\circ}$ and $^{\circ}$ the ${ }^{\circ}$ calibrant $^{\circ}[9] .{ }^{\circ}$ With an increasing concentration of the target compound at a fixed concentration of the calibrant, the measured difference in $\mathrm{m} / \mathrm{z}$ increased as that of the analyte shifted to a lower value compared to that of the calibrant. A decreasing concentration of target gave, on the other hand, a decreasing difference between the $\mathrm{m} / \mathrm{z}$ values of the analyte and its internal standard. Several factors contribute to these mass inaccuracies on a TOF. When the ion number is small, errors due to statistics cause deterioration of peak shape, whereas when the ion number is large, peak shapes become distorted owing to coincident ion strikes and dead-time issues. When the target and internal calibrant are 1:1, deviations in peak shape are canceled, and the mass accuracy was $1.0 \pm 0.1 \mathrm{ppm}$ for three determinations. With six determinations, we found an accuracy of $0.3 \pm 0.5 \mathrm{ppm}$. Achieving a 1:1 ratio in real analyses is possible but

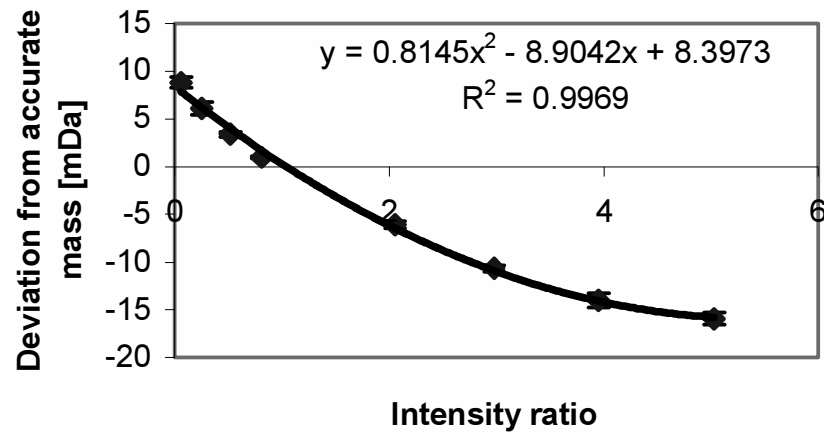

Figure 1. Correction function for the shift in accurate mass with signal intensity ratios between caffeine (target compound) and ${ }^{13} \mathrm{C}_{3}$-caffeine (mass calibrant).

requires reanalysis after adjusting concentrations based on a "scout" run.

When the concentration ratios deviate from unity but remain in an acceptable range for quantification, the mass measurement errors increase to as high as 15 millimass units $^{\circ}$ (Figure $\left.{ }^{\circ} 1\right){ }^{\circ}{ }^{\circ} \mathrm{We}^{\circ}$ found ${ }^{\circ}{ }^{\text {we }}{ }^{\circ}$ could $^{\circ}{ }^{\circ}$ compensate ${ }^{\circ}$ for the errors by invoking a correction function (experimentally determined to be quadratic) that corrects the measured mass ${ }^{\circ} s^{\circ}$ afunction ${ }^{\circ} f^{\circ}$ signal $^{\circ}$ intensity ${ }^{\circ}$ atios $^{\circ}$ (Figure 1 ). The best fit has an $R^{2}$ of 0.9969 whereas a linear fit gives an $R^{2}$ of 0.9553 .

The absolute number of ion counts also influences mass accuracy. The data acquisition for the standard curve and the samples should be done under conditions that have similar ion counts. The optimal number of counts for mass accuracy as recommended by Micromass for the QTOF is 200-400. For the calibration curve, the counts must vary more: for caffeine, 1004000 counts/s, and for ${ }^{13} \mathrm{C}_{3}$-caffeine, $800-1500$ at chromatographic peak tops. Lower maximum counts gave a poorer dynamic range and more scatter of the correction plot. When applied to the test samples, the counts for caffeine and its isotopomer were within range (i.e., 500-4000 and 800-1400 counts/s, respectively).

Ion counts vary widely from valley to chromatographic peak top. Further, a sharp, high chromatographic peak will result in fewer spectra with higher absolute intensity than a broad, flat peak. To stay within the recommended count range, one may sum spectra at the edges and exclude count-rich scans at the peak top. To be consistent and avoid time-consuming manual inspection, however, all spectra of a chromatographic peak were summed and used for a single correction scheme. Although this gave higher errors at the extremes of concentration ratios, it did provide better reproducibility, less scatter, and better ion statistics.

To test the correction procedure, three caffeine samples were run in triplicate. The caffeine concentrations were 60,120 , and $350 \mathrm{nM}$, and the ${ }^{13} \mathrm{C}_{3}$-caffeine was 100-nM. The error in accurate mass was calculated from a previously established correction function and subtracted from the measured mass. The corrected results show an average deviation of $-2 \pm 3 \mathrm{ppm}(\mathrm{N}=9)$, 
which is a significant improvement over the accuracies achieved without the correction.

\section{Conclusions}

Although the concentration range for measuring accurate mass with a Q-TOF is limited to approximately a factor of 20, a mass correction, when done under proper conditions, affords high accuracy at low detection limits while diminishing the need for repeat analyses. The detection limit is better than those reported by others using ${ }^{\circ} \mathrm{LC}-\mathrm{MS} / \mathrm{MS}^{\circ}[13]^{\circ}$ and $^{\circ}$ yet $^{\circ}$ the ${ }^{\circ}$ specificity ${ }^{\circ}$ is ${ }^{\circ}$ high owing to the accurate-mass measurement. We found the limit of detection to be one order of magnitude better in the accurate-mass versus the MS/MS mode. Furthermore, the good chromatographic resolution and stable retention times afforded by capillary LC added to the certainty. It is possible that improved mass accuracy over a larger dynamic range can be achieved with LC/FTMS, and future plans call for an evaluation of this prospect.

\section{Acknowledgments}

This work was supported by the National Institutes of Health grant nos. P41RR00954 and 1P01CA49210.

\section{References}

1. Eljarrat, E.; Barcelo, D. Congener-specific determination of dioxions and related compounds by gas chromatography coupled to LRMS, HRMS, MS/MS, and TOFMS. J. Mass. Spectrom. 2002, 37, 1105-1117.

2. Cai, Z.; Giblin, D. E.; Ramanujam, V. M. S.; Gross, M. L. Mass-profile monitoring in trace analysis: Identification of polychlorodibenzothiophenes in crab tissue collected from the Newark/Raritan bay system. Environ. Sci. Tech. 1994, 28, 1535-1538.

3. Gross, M. L.; Sun, T.; Lyon, P. A.; Wojinski, S. F.; Hilker, D. R.; Dupuy, A. E., Jr.; Heath, R. G. Method validation for the determination of tetrachlorodibenzodioxin at the low partsper-trillion level. Anal. Chem. 1981, 53, 1902-1906.
4. Tong, H. Y.; Giblin, D. E.; Lapp, R. L.; Monson, S. J.; Gross, M. L. Mass profile monitoring in trace analysis by gas chromatography/mass spectrometry. Anal. Chem. 1991, 63, 17721780.

5. Gross, M. L. Application of mass spectrometric methods to analysis of xenobiotics in biological systems; Vol. 39. IARC Scientific Publications: Lyon, 1982.

6. Bristow, A. W. T.; Webb, K. S. Intercomparison study on accurate mass measurement of small molecules in mass spectrometry. J. Am. Soc. Mass Spectrom. 2003, 14, 1086-1098.

7. Hau, J.; Stadler, R.; Jenny, T. A.; Fay, L. B. Tandem mass spectrometric accurate mass performance of time-of-flight and Fourier transform ion cyclotron resonance mass spectrometry: A case study with pyridine derivatives. Rapid Commun. Mass Spectrom. 2001, 15, 1840-1848.

8. Clauwaert, K.; Vande Casteele, S.; Sinnaeve, B.; Deforce, D.; Lambert, W.; Van Peteghem, C.; Van Bocxlaer, J. Exact mass measurement of product ions for the structural confirmation and identification of unknown compounds using a quadrupole time-of-flight spectrometer: A simplified approach using combined tandem mass spectrometric functions. Rapid Commun. Mass Spectrom. 2003, 17, 1443-1448.

9. Blom, K. F. Estimating the precision of exact mass measurements on an orthogonal time-of-flight mass spectrometer. Anal. Chem. 2001, 73, 715-719.

10. Wolff, J.-C.; Eckers, C.; Sage, A. B.; Giles, K.; Bateman, R. Accurate mass liquid chromatography/mass spectrometry on quadrupole orthogonal acceleration time-of-flight mass analyzers using switching between separate sample and reference sprays. 2. Applications using the dual-electrospray ion source. Anal. Chem. 2001, 73, 2605-2612.

11. Eckers, C.; Wolff, J.-C.; Haskins, N. J.; Sage, A. B.; Giles, K.; Bateman, R. Accurate mass liquid chromatography/mass spectrometry on orthogonal acceleration time-of-flight mass analyzers using switching between separate sample and reference sprays. 1. Proof of concept. Anal. Chem. 2000, 72, 3683-3688.

12. Zhang, N.; Fountain, S. T.; Bi, H.; Rossi, D. T. Quantification and rapid metabolite identification in drug discovery using API time-of-flight LC/MS. Anal. Chem. 2000, 72, 800-806.

13. Schneider, H.; Ma, L.; Glatt, H. Extractionless method for the determination of urinary caffeine metabolites using highperformance liquid chromatography coupled with tandem mass spectrometry. J. Chromatogr. B 2003, 789, 227-237. 